\title{
Mundo virtual Minecraft: uma Experiência no Ensino de Circuitos Digitais
}

\author{
Henry E. L. Cagnini ${ }^{1}$, Andrea S. Charão ${ }^{2}$, \\ Patrícia P. de A. Barcelos ${ }^{2}$, Bruno R. de Azevedo ${ }^{3}$ \\ ${ }^{1}$ Programa de Pós-Graduação em Ciência da Computação \\ Pontifícia Universidade Católica do Rio Grande do Sul \\ Porto Alegre, RS, Brasil \\ ${ }^{2}$ Departamento de Linguagens e Sistemas de Computação \\ Universidade Federal de Santa Maria \\ Santa Maria, RS, Brasil \\ ${ }^{3}$ Programa de Pós-Graduação em Informática \\ Universidade Federal de Santa Maria \\ Santa Maria, RS, Brasil
}

henry.cagninieacad.pucrs.br, \{andrea, pitthan, brunodea\}einf.ufsm.br

\begin{abstract}
Resumo. Mundos virtuais têm proporcionado novas experiências no processo de ensino-aprendizagem. Um exemplo de mundo virtual é o do jogo Minecraft, que oferece entretenimento a milhões de usuários e vem despertando o interesse de educadores. Neste trabalho, apresenta-se uma experiência de uso do Minecraft na disciplina de Circuitos Digitais, em uma instituição de ensino superior. Para isso, construiu-se um conjunto de desafios inseridos no mundo virtual, utilizando conceitos de portas lógicas e multiplexadores. Os desafios foram propostos a alunos calouros de Ciência da Computação e Sistemas de Informação. A experiência permitiu levantar impressões, tanto positivas como negativas, sobre a aplicabilidade do Minecraft neste contexto.
\end{abstract}

\begin{abstract}
Virtual worlds have provided new experiences in the teachinglearning process. A virtual world example is the Minecraft game, which provides entertainment to millions of users and is attracting the interest of educators. In this paper, we present an experience on using Minecraft in a Digital Circuits course in higher education. To this end, we built a set of challenges inserted in the virtual world, using concepts of logic gates and multiplexers. We propose those challenges to freshmen students in Computer Science and Information Systems. The experience results in some impressions, both positive and negative, on the applicability of Minecraft in this context.
\end{abstract}

\section{Introdução}

Com a popularização de dispositivos eletrônicos, tais como celulares e computadores, o uso de software é frequente nos mais variados públicos e esferas sociais. O público jovem, em particular, adere facilmente a novas tecnologias, tornando-se usuário dos mais variados tipos de software. Para este público, é atrativa a utilização de novas abordagens pedagógicas mediadas por software, tanto aqueles construídos com propósitos educacionais como os de uso geral que se revelam úteis a esse propósito. 
Os mundos virtuais, ou MVs, são um tipo de software, educacional ou de entretenimento, cuja principal característica é prover uma experiência imersiva ao usuário, através da emulação do mundo real. Mundos virtuais têm proporcionado novas experiências no processo de ensino-aprendizagem, inclusive no ensino superior [de Freitas e Neumann 2009]. Os mundos virtuais Second Life e OpenSimulator são exemplos bastante usados nesse contexto [Esteves et al. 2011].

No ramo do entretenimento, dentre os jogos que se baseiam em mundos virtuais, destaca-se o jogo Minecraft, que conquistou milhões de usuários nos últimos anos [McVeigh 2013]. Embora Minecraft não tenha sido concebido com propósitos educacionais, seu mundo virtual tem despertado o interesse de educadores, por permitir grande liberdade de criação e proporcionar experiências variadas. Suas aplicações no ensino incluem, por exemplo, tópicos em matemática, história, música e computação [TeacherGaming 2014].

Motivado por este cenário, o principal objetivo deste trabalho foi explorar o uso do Minecraft no ensino superior em computação, aplicando-o a uma disciplina oferecida para calouros, em que reprovações são frequentes. A disciplina escolhida foi a de Circuitos Digitais, ofertada no primeiro semestre dos cursos de Ciência da Computação e Sistemas de Informação da Universidade Federal de Santa Maria (UFSM), no RS.

O artigo é organizado da seguinte forma: na seção 2 aborda-se o conceito de mundos virtuais e, na seção 3, apresenta-se Minecraft em mais detalhes. Na seção 4, discute-se trabalhos relacionados que exploram mundos virtuais no ensino. A seção 5 apresenta o desenvolvimento da experiência e seu método de avaliação. Na sequência, as seções 6 e 7 apresentam e discutem os resultados obtidos. A seção 8, por fim, apresenta considerações finais sobre o trabalho.

\section{Mundos Virtuais}

Mundos virtuais (MVs) podem ser caracterizados como ambientes criados por computador, pelos quais o usuário movimenta o avatar que o representa, podendo interagir com o ambiente e com avatares de outros usuários [Nelson e Erlandson 2012]. Originalmente utilizados em jogos recreacionais e aplicações de simulação, os mundos virtuais são atualmente vislumbrados também como ferramentas de socialização e educação [Rafalski et al. 2014].

Quando utilizados no ensino, MVs encaixam-se no modelo de educação construtivista: o aluno (jogador, agente ativo do MV) adquire o conhecimento de forma experimental, através de atividades exercidas sobre objetos do mundo virtual [Rafalski et al. 2014]. Cabe então ao tutor, construtor das atividades dentro do mundo virtual, prover atividades tanto interessantes como desafiadoras aos alunos, de forma que estes possam se apropriar do conteúdo e levá-lo para a vida real [Strommen e Lincoln 1992].

São exemplos de MVs as ferramentas Second Life ${ }^{1}$ e OpenSimulator ${ }^{2}$. O Second Life é um mundo virtual 3D lançado em 2003, pela Linden Lab, sediada em San Francisco, EUA, voltado principalmente para a interação social em um ambiente que simula a vida real. Já o OpenSimulator é uma ferramenta open source escrita em linguagem

\footnotetext{
${ }^{1}$ Disponível em https://secondlife.com

${ }^{2}$ Disponível em http://opensimulator.org
} 
C\#, criada em 2007, por meio da qual podem ser construídos mundos virtuais interconectáveis. Ambos os MVs suportam interação online em ambientes 3D e permitem a criação de conteúdos em tempo real. Outros exemplos de mundos virtuais também são encontrados em jogos, como é o caso do Minecraft, apresentado na seção a seguir.

\section{Minecraft}

Minecraft é um jogo produzido pelo estúdio sueco Mojang e lançado em 2009. O jogo segue o estilo sandbox - isto é, o jogador não é impelido a perseguir um objetivo principal, ficando livre para realizar quaisquer atividades dentro do jogo. Minecraft divide-se em dois modos de jogo principais: creative e survival. No modo creative, não existe limite de recursos que podem ser utilizados pelo jogador. Já o modo survival impõe restrições, tais como a necessidade de se alimentar e construir abrigo para se proteger de inimigos.

Além de ser um jogo sandbox, Minecraft é também um mundo virtual. É importante salientar que estas duas categorias de jogos são distintas, podendo ou não haver intersecção entre elas. Pelo fato de Minecraft ser um jogo sandbox, a flexibilidade de utilizá-lo para tarefas educacionais é maior em comparação a outros gêneros de jogos. De fato, as restrições impostas pelo jogo são poucas, permitindo a posterior adição de restrições por um tutor ou professor sem que isso torne o jogo desinteressante na visão do aluno.

Minecraft é também um jogo aberto ao modding - capacidade de modificar aspectos básicos do jogo por terceiros. Um projeto que modificou Minecraft com propósitos educacionais é o MinecraftEdu [TeacherGaming 2014]. Esta modificação visa facilitar o ensino de diversas áreas de conhecimento, desde humanidades (como história e geografia) até computação.

\section{Mundos Virtuais no Ensino}

O uso de mundos virtuais no ensino é tema de muitos trabalhos. O Second Life, como um agente precursor na popularização de MVs, há muitos anos é alvo de pesquisas e práticas em diferentes níveis de ensino, desde a educação básica [da Cunha et al. 2008] até o ensino superior [Jarmon et al. 2009, Esteves et al. 2011], seja em atividades presenciais como no ensino à distância [Sousa et al. 2009, Zanotto et al. 2009, Inman et al. 2010]. O OpenSim, por sua vez, tem sido empregado em trabalhos mais recentes, como ferramenta facilitadora para construção de novos mundos virtuais voltados para o ensino [Allison et al. 2012, Voss et al. 2014, Nunes et al. 2014].

Especificamente no ensino superior em computação, há relatos de experiências bem sucedidas com mundos virtuais em tópicos como, por exemplo, lógica e programação [Esteves et al. 2011], engenharia de software [Rodrigues et al. 2013] e redes de computadores [Voss et al. 2014].

O jogo Minecraft, por sua vez, tem no projeto MinecraftEdu um incentivo ao seu uso no ensino. No entanto, na literatura acadêmica e científica brasileira, percebe-se que ainda há poucas experiências com o MV oferecido por este jogo. Em [Fontes 2012], Minecraft é utilizado sem nenhuma modificação para o ensino de história em uma escola técnica. Verificou-se, neste trabalho, que os alunos inicialmente desejaram jogar Minecraft apenas para entreter-se mas, com o incentivo da professora, metade da turma convergiu para a realização da atividade proposta (construção de um castelo medieval). Em 
[Dias e Rosalen 2014], elaborou-se uma experiência utilizando MinecraftEdu para alunos do ensino fundamental, na disciplina de biologia. Percebeu-se que os alunos primeiramente fugiram da realização da atividade, porém com o tempo foram retornado aos desafios e realizando-os em colaboração com os outros alunos. No ensino superior, o uso do MV Minecraft parece ser ainda mais incipiente, conforme estudos em [Martinez 2014].

No que diz respeito ao ensino de circuitos digitais, realizou-se uma busca em conferências que relacionam educação e computação (ACM Technical Symposium on Computer Science Education - SIGCSE, Workshop sobre Educação em Computação WEI e Simpósio Brasileiro de Informática na Educação - SBIE) nos últimos 5 anos, mas não encontrou-se trabalhos relacionando esta área a mundos virtuais. No WIE, em particular, foram encontrados trabalhos voltados ao ensino de circuitos digitais apenas em edições mais antigas [Pereira et al. 2006, Sena e Torres 2009], porém usando outras ferramentas educacionais. Sabe-se, no entanto, da importância desta disciplina para cursos de graduação na área de computação e, também, que existem recursos associando o MV Minecraft a assuntos dessa disciplina ${ }^{3}$. Este cenário, embora não esteja amparado em uma pesquisa exaustiva, reforçou a motivação para o presente trabalho.

\section{Minecraft na Disciplina de Circuitos Digitais}

Para aplicar o MV Minecraft na disciplina de Circuitos Digitais, construiu-se um conjunto de desafios reunidos em um "mapa". Na terminologia do jogo, um mapa é um mundo virtual personalizado, compreendendo paisagem, estruturas (prédios, casas, salas, muros, etc.) e, possivelmente, placas e anotações com informações para o usuário. No mapa criado, os desafios estão dispostos sobre estruturas de forma sequencial, de modo que a solução de um desafio habilita o acesso ao desafio/estrutura subsequente. Cada desafio exige a utilização de conhecimentos adquiridos em aulas teóricas de Circuitos Digitais, em nível crescente de dificuldade, conforme o programa da disciplina ${ }^{4}$. A relação entre os desafios e os tópicos é apresentada na Tabela 1. Os desafios 1 e 2 usam intensivamente os recursos de placas e anotações no mapa, enquando os demais envolvem objetos e estruturas dispostos na paisagem. A representação de portas lógicas no jogo foi inspirada em tutoriais da comunidade de jogadores ${ }^{3}$. O mapa construído está disponível em: https://sites.google.com/a/inf.ufsm.br/minecraft-circdig/.

\begin{tabular}{l|l}
\hline Desafio & Tópico \\
\hline 1) Sair da casa & familiarização com cenário e controles do jogo \\
2) Tutorial básico & revisão de conceitos de circuitos digitais \\
3) Entrada da caverna & valores de variáveis \\
4) Sensor de luz & portas AND e NOR \\
5) Vaca vermelha & porta NAND \\
6) Rio de lava & porta XOR \\
7) Vilarejo subterrâneo & multiplexador de duas entradas e uma chave seletora \\
\hline
\end{tabular}

Tabela 1. Desafios e seus respectivos tópicos

O mapa criado foi aplicado para a turma da disciplina de Circuitos Digitais, no segundo semestre de 2014. De 40 matriculados, 25 estiveram presentes para realizar a

\footnotetext{
${ }^{3}$ Disponíveis em: http://minecraft.gamepedia.com/Logic_circuit

${ }^{4}$ Disponível em: http://www.inf.ufsm.br
} 
atividade, que foi aplicada na segunda metade do semestre. A maioria dos alunos cursava a disciplina pela primeira vez no curso de Sistemas de Informação, que tem ingresso no segundo semestre, mas havia também alunos repetentes de Ciência da Computação ou Sistemas de Informação. Um dos tópicos (multiplexadores, no desafio 7) ainda não havia sido visto pela turma, permitindo verificar o comportamento dos alunos frente a um assunto desconhecido, mas fortemente relacionado. A atividade foi proposta aos alunos como um exercício, não havendo pontuação associada na avaliação formal da disciplina. Para aplicação dos desafios, em laboratório, contou-se com o auxílio de 5 monitores voluntários, jogadores habituais de Minecraft, que já haviam cursado a disciplina. Durante a realização da atividade, os tutores circulavam pelo laboratório auxiliando os alunos com quaisquer dificuldades que pudessem surgir, como ilustra a Figura 1.

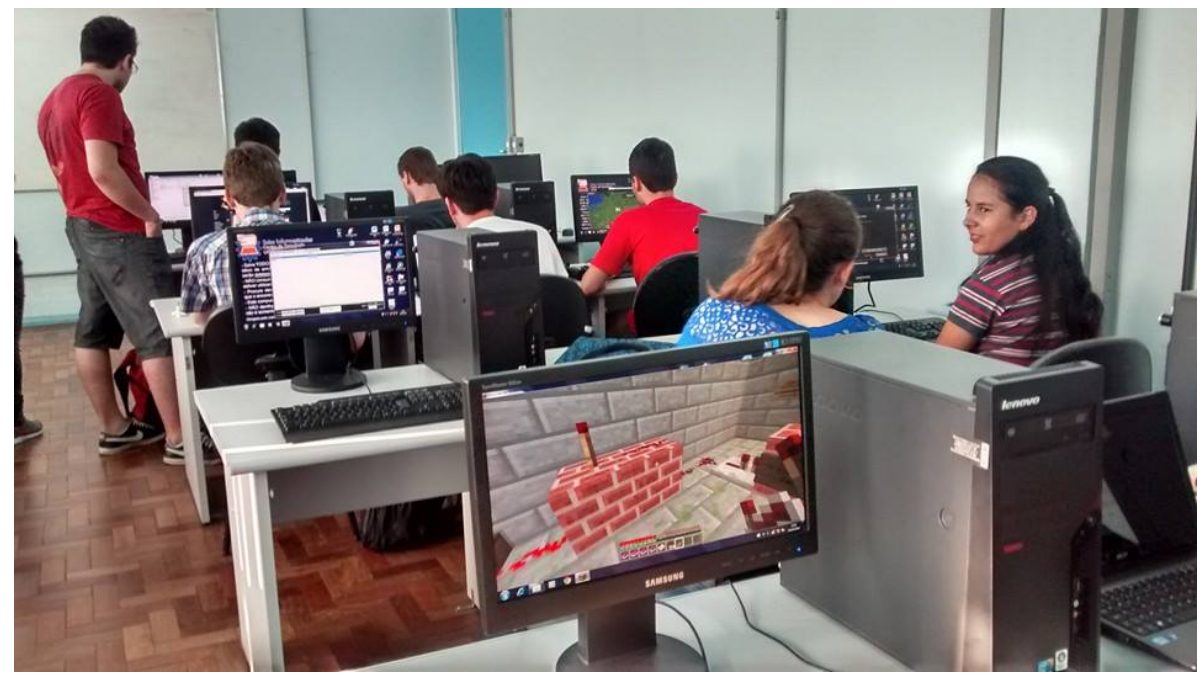

Figura 1. Imagem obtida durante a realização da atividade

Para avaliar a experiência, elaborou-se um questionário que foi aplicado aos alunos ao final da atividade, visando coletar suas impressões acerca de diversos aspectos. $\mathrm{O}$ preenchimento não era obrigatório e todas as questões, com exceção da última, eram de múltipla escolha. As questões usavam um linguajar informal, aproximando-se da linguagem utilizada pelos alunos no cotidiano, sendo transcritas literalmente a seguir:

- Você já tinha ouvido falar sobre Minecraft?

- Com que frequência você joga Minecraft?

- O que você achou dessa atividade?

- Marque a dificuldade que você encontrou em cada uma das etapas dessa atividade.

- Quais desses tópicos você identificou como presentes nessa atividade?

- O quanto você acha que aprendeu sobre os seguintes tópicos, depois de realizar essa atividade?

- Qual das seguintes atividades você acha melhor para cada uma das seguintes disciplinas?

- Alguma sugestão ou reclamação?

Para analisar as respostas dos alunos, construiu-se um dataset - conjunto de dados, onde as perguntas são agrupadas como colunas e a resposta de cada aluno como uma linha - tendo como atributo classe - a informação mais importante do dataset - a pergunta " $O$ 
que você achou dessa atividade?". O dataset possibilitou o cruzamento de informações, permitindo uma análise detalhada de impressões dos alunos.

\section{Resultados Obtidos}

Pôde-se perceber, logo no início da experiência, que alguns alunos não realizaram as atividades prontamente, vagando pelo mundo virtual e explorando seus aspectos e controles. Apenas depois de um tempo estes alunos se engajaram na resolução dos desafios. Muitos alunos recorreram ao auxílio dos voluntários quando encontraram dificuldades de operação do jogo ou de compreensão e raciocínio para completar um desafio.

Ao final, foram coletadas 23 respostas ao questionário. Na Figura 2 apresenta-se as respostas dos alunos à pergunta "O que você achou dessa atividade?", que revela a percepção geral dos alunos sobre a experiência. Nota-se que, em sua maioria, os alunos tiveram uma impressão positiva ("Legal" ou "Muito Legal").

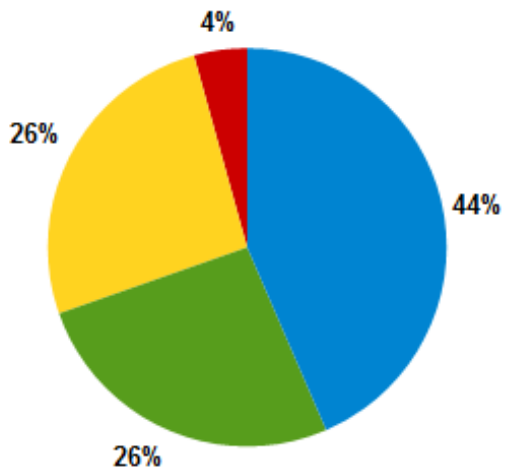

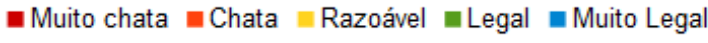

\section{Figura 2. Respostas para a pergunta "O que você achou dessa atividade?"}

A percepção do aluno pode depender de alguns fatores, tais como a familiaridade com o jogo e dificuldade imposta pela atividade. Na Figura 3, relaciona-se a percepção do aluno à frequência com que ele joga Minecraft fora da sala de aula. Percebe-se que a avaliação regular a negativa é maior pelos alunos que nunca tiveram contato com Minecraft, e melhora à medida que contato é mais frequente.

Quanto à dificuldade da atividade, vale lembrar que os desafios foram encadeados de forma crescente, desde níveis iniciais com várias explicações até um tópico ainda não visto na disciplina. A Figura 4 relaciona a dificuldade percebida pelos alunos com aquela planejada para cada desafio. Verifica-se, de forma geral, que os alunos percebem os desafios iniciais como mais fáceis e os finais como mais difíceis. No entanto, notou-se efeitos não previstos: vários alunos desistiram dos desafios e, além disso, as dificuldades começaram a ser significativas a partir do quarto desafio, com muitos alunos atribuindo "Médio" ou "Difícil" a desafios planejados para serem relativamente fáceis. Notou-se que os alunos que iam desistindo de vencer os desafios acabavam por jogar Minecraft no modo survival, coletando recursos para a sobrevivência dentro do jogo.

Apesar da fuga da atividade por parte dos alunos, muitos julgaram o uso de mundo virtual melhor do que outras atividades para a disciplina em questão. Isso pode ser deduzido da Figura 5, que relaciona a percepção geral da atividade com o método de ensino 


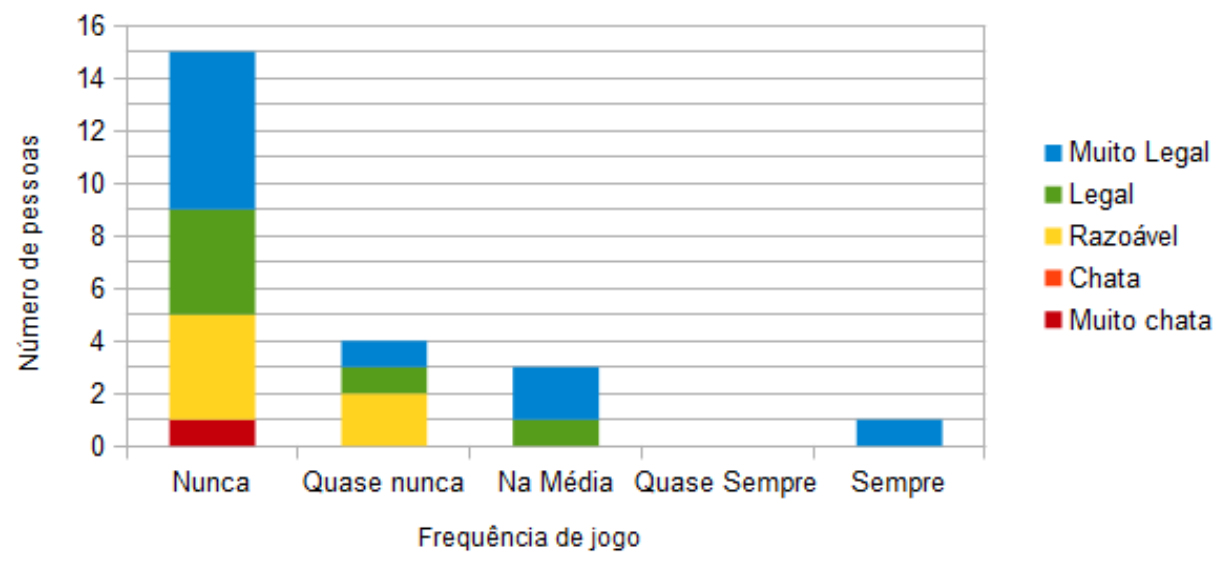

Figura 3. Frequência de uso de Minecraft versus a percepção do aluno sobre a atividade

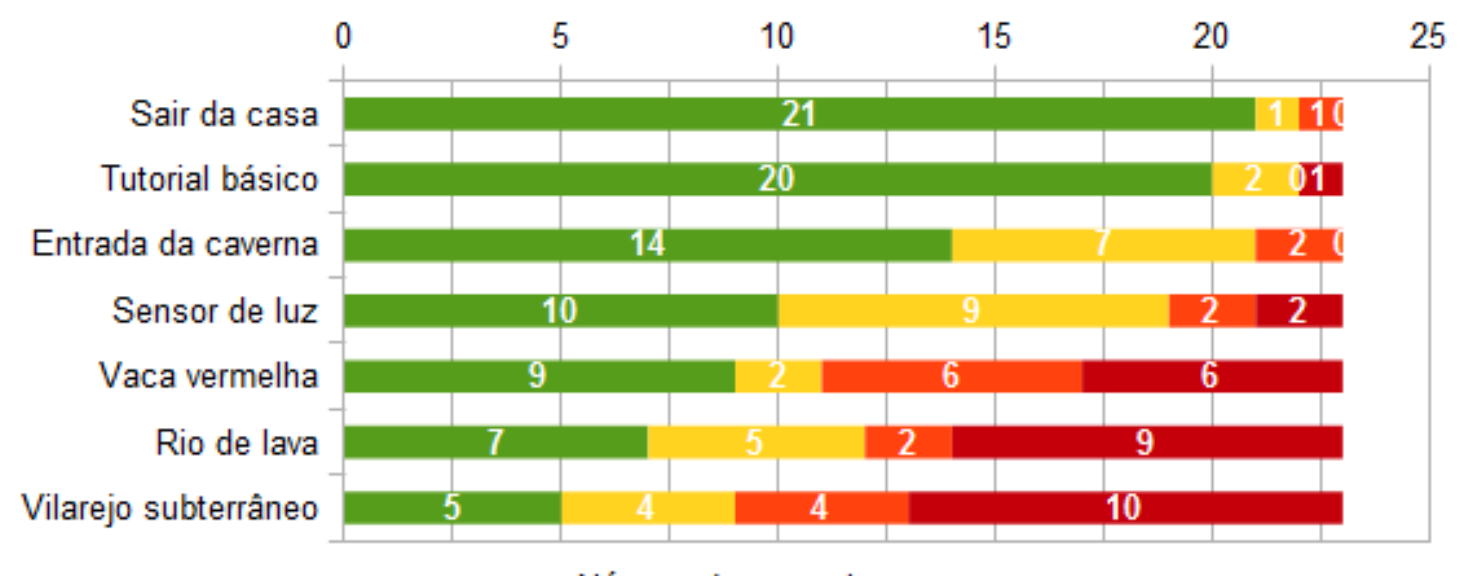

Número de respostas

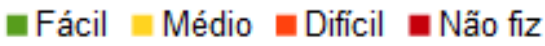

Figura 4. Dificuldade percebida em cada um dos desafios propostos. Os desafios estão em ordem crescente de dificuldade planejada, com o mais fácil no topo e o mais difícil na base.

preferencial para aprender circuitos digitais. Como esperado, nota-se que a preferência por atividades tradicionais é maior por parte dos alunos que não consideraram a atividade "Legal" ou "Muito Legal".

\section{Discussão}

As impressões positivas sobre a atividade reforçaram os resultados já verificados em outros trabalhos relacionados. Mesmo assim, há aspectos que merecem mais atenção e admitem melhorias, conforme discutido nos parágrafos a seguir.

- Limitação de área e controles: Em alguns desafios, os alunos podiam se locomover livremente para explorar o mundo virtual, como ocorre tipicamente nos principais modos de jogo em Minecraft. Dados os objetivos dos desafios, e contando com a ação dos monitores, esperava-se pouca locomoção exploratória. No 


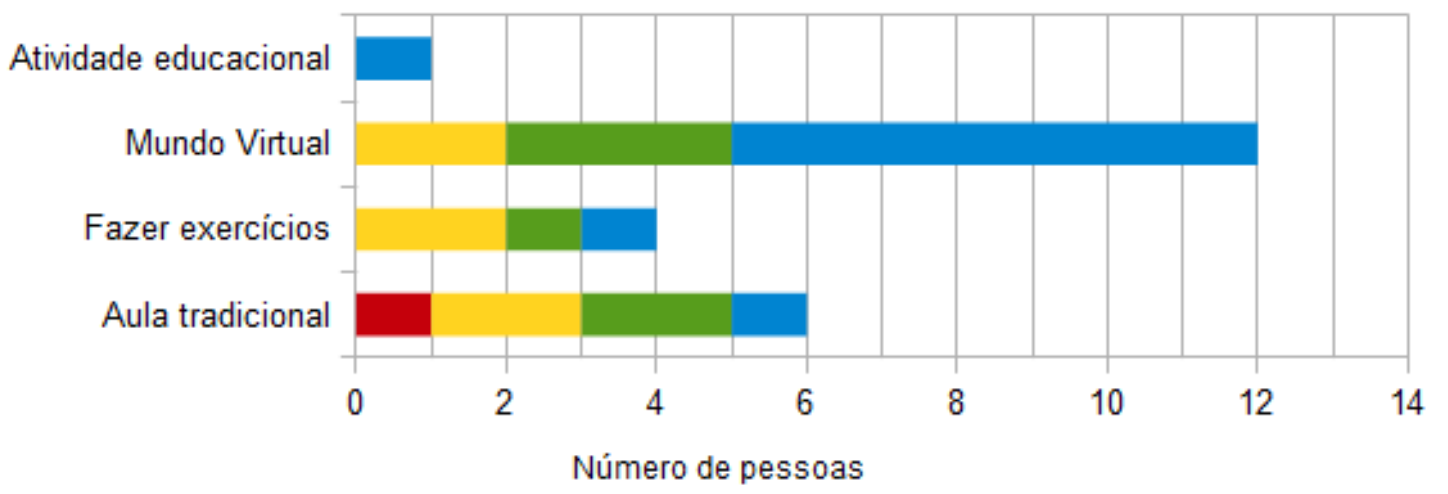

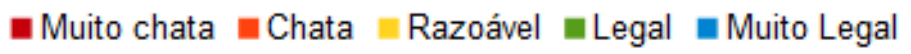

Figura 5. Respostas para a pergunta "Qual das seguintes atividades você acha melhor para a disciplina de Circuitos Digitais?", agrupadas pela percepção geral da atividade.

entanto, observou-se diversos alunos deixando a área destinada aos desafios. Diante disso, propõe-se limitar mais rigidamente, através de muros virtuais, a área de locomoção dos alunos. Adicionalmente, para evitar que usuários mais experientes no jogo contornem a limitação de área (por exemplo, destruindo muros), recomenda-se desabilitar o uso de cheats, i.e., códigos que permitem ao usuário alterar aspectos do jogo enquanto estiver jogando. Isso pode ser configurado nas "Opções de Mundo", quando se cria um novo mapa no Minecraft.

- Curva de aprendizado: Os desafios propostos foram concebidos para revelar dificuldade crescente. Pôde-se notar que desafios mais complexos têm potencial de desestimular o aluno, com no caso do último desafio, que foi realizado por apenas $56 \%$ dos alunos. Para suavizar a curva de aprendizado (levando em conta a ferramenta e também os tópicos da disciplina), percebe-se a necessidade de elaborar-se mais desafios simples e intermediários. Alunos mais experientes no jogo poderão mesmo assim avançar rapidamente, enquanto o restante poderá progredir de forma confortável. Além disso, dos $56 \%$ dos alunos que concluíram o último desafio, $62 \%$ classificaram-no com dificuldade média a difícil, e apenas $38 \%$ o consideraram fácil. Este foi o único desafio em que a quantidade de alunos que percebeu a dificuldade média ou difícil superou os que a perceberam como fácil (vide Fig. 4). Também foi este o único desafio que abordou um tema não visto em outras aulas, portanto entende-se que o mundo virtual Minecraft deva ser usado cautelosamente, de forma a reforçar outros métodos tradicionais de ensino, mesmo que o jogo tenha a preferência dos alunos.

- Atuação dos monitores: Entende-se que a ação dos 5 monitores foi determinante para o sucesso da experiência, pois eles foram bastante requisitados pelos alunos durante a atividade. Todos os monitores foram voluntários e, nesse sentido, observou-se que eles se mostraram facilmente motivados a participar, dado que conheciam o Minecraft em outro contexto (entretenimento). Colaborando para o uso do Minecraft numa disciplina, os monitores também reforçaram seus conhecimentos em circuitos digitais e trocaram experiências entre si e com os mais 
jovens, o que caracteriza um efeito colateral muito bem-vindo para a experiência realizada. Em trabalhos relacionados, não foram encontradas observações sobre este aspecto.

\section{Considerações Finais}

Este trabalho abordou a realização de uma atividade utilizando o mundo virtual Minecraft para ensino de circuitos digitais. A atividade foi bem aceita pelos alunos, que em sua maioria classificaram a atividade como positiva. A experiência reforçou impressões sobre a aplicabilidade deste MV no ensino e, adicionalmente, permitiu levantar aspectos que podem ser melhorados para que os alunos não se dispersem no mundo virtual e tenham uma curva de aprendizado mais suave. Por fim, notou-se um efeito colateral positivo com relação à atuação dos monitores, dada sua experiência e motivação no uso de Minecraft em entretenimento. A experiência não foi exaustiva, mas concentrou-se em uma ferramenta ainda pouco explorada em estudos voltados ao ensino superior. Neste sentido, incentiva-se a reprodução da experiência em outros momentos, para o que disponibiliza-se o mapa Minecraft elaborado.

\section{Agradecimentos}

Agradecemos ao professor Dr. Everton Alceu Carara, pelo tempo concedido para a realização da atividade com sua turma de Circuitos Digitais em 2014, e a todos os monitores, membros do projeto "Clube de Computação", que colaboraram voluntariamente e prestaram um auxílio de grande valor: Cassiano A. D. da S. Schneider, Érica S. de Lima, Eduardo L. Lemos, Guilherme F. Hoffmann e Matuzalem da C. Borges.

\section{Referências}

Allison, C., Campbell, A., e et al, C. J. D. (2012). Growing the use of Virtual Worlds in education: an OpenSim perspective. In Proceedings of the 2nd European Immersive Education Summit (EiED '12).

da Cunha, P. F. V., Giraffa, L. M. M., Greis, L. K., e Portanova, R. (2008). Second Life como recurso para o ensino da Matemática. In Anais do Simpósio Brasileiro de Informática na Educação.

de Freitas, S. e Neumann, T. (2009). The use of 'exploratory learning' for supporting immersive learning in virtual environments. Comput. Educ., 52(2):343-352.

Dias, N. F. e Rosalen, M. (2014). Minecraft: Uma estratégia de ensino para aprender mais jogando. In Anais do Simpósio Internacional de Educação a Distância.

Esteves, M., Fonseca, B., Morgado, L., e Martins, P. (2011). Improving teaching and learning of computer programming through the use of the Second Life virtual world. British Journal of Educational Technology, 42(4):624-637.

Fontes, F. G. (2012). Jogando Minecraft na aula de história. In Anais do Simpósio do Ensino Médio e Técnico, pages 34-36. Centro Paula Souza.

Inman, C., Wright, V. H., e Hartman, J. A. (2010). Use of Second Life in K-12 and Higher Education: A Review of Research. Journal of Interactive Online Learning, 9(1). 
Jarmon, L., Traphagan, T., Mayrath, M., e Trivedi, A. (2009). Virtual world teaching, experiential learning, and assessment: An interdisciplinary communication course in Second Life. Computers \& Education, (53):169-182.

Martinez, S. G. (2014). Using Commercial Games to Support Teaching in Higher Education. $\mathrm{PhD}$ thesis, Concordia University, Canada.

McVeigh, T. (2013). Minecraft: how a game with no rules changed the rules of the game for ever. The Guardian.

Nelson, B. e Erlandson, B. (2012). Design for Learning in Virtual Worlds. Interdisciplinary approaches to educational technology. Routledge.

Nunes, F. B., Herpich, F., Voss, G. B., Medina, R. D., de Lima, J. V., e Tarouco, L. M. R. (2014). Laboratório Virtual de Química: uma ferramenta de estímulo à prática de exercícios baseada no Mundo Virtual OpenSim. In Anais do Simpósio Brasileiro de Informática na Educação.

Pereira, M. C., da Silva Martins, P., Batista, E. A., da Silva, A. C. R., e Leonardi, F. (2006). Motivando Alunos em Circuitos Digitais: um Estudo de Caso. In Anais do Workshop sobre Educação em Computação, pages 188 - 195.

Rafalski, J. P., Júnior, R. R. M. V., e da Silva, C. A. S. (2014). Mundos Virtuais como Suporte à Aprendizagem - Uma Avaliação na Implementação de Projetos de Aprendizagem. Revista Novas tecnologias na educação, 12(1).

Rodrigues, G., Soria, A., e Campo, M. (2013). Virtual Scrum: A teaching aid to introduce undergraduate software engineering students to Scrum. Computer Applications in Engineering Education, 23(1).

Sena, A. e Torres, M. (2009). EasyKarnaugh 3.0 - Uma ferramenta computacional para o auxílio no ensino de Mapas de Karnaugh em Lógica Digital. In Anais do Workshop sobre Educação em Computação, pages 455 - 464.

Sousa, P. M. S., Silva, W. B., Rolim, C., Bessa, W., e Fernandes, S. (2009). O uso do Second Life na construção de um ambiente virtual de aprendizagem. In Anais do Workshop sobre Educação em Computação, pages 587 - 590.

Strommen, E. F. e Lincoln, B. (1992). Constructivism, Technology, and the future of classroom learning. Education and Urban Society, 24(4):466-476.

TeacherGaming (2014). MinecraftEdu. Disponível em: http://minecraftedu.com.

Voss, G. B., Oliveira, V., Nunes, F. B., Herpich, F., Medina, R. D., e Bercht, M. (2014). Construção e Análise de um Mundo Virtual 3D para o Ensino e Aprendizagem de Redes de Computadores. In Anais do Simpósio Brasileiro de Informática na Educação.

Zanotto, D., Rolim, C., e Fernandes, S. (2009). Desenvolvimento de um SurveyBot em Mundos Virtuais com Aplicação em Sistemas de Avaliação. In Anais do Workshop sobre Educação em Computação, pages 623 - 626. 